\title{
Majorana Bound States and Zero-Bias Conductance Peaks in Superconductor/Semiconductor Nanowire Devices
}

\author{
A. KobiatKA ${ }^{a}$ And A. PTOK ${ }^{b, *}$ \\ ${ }^{a}$ Institute of Physics, Maria Curie-Skłodowska University, \\ Plac Marii Skłodowskiej-Curie 1, PL-20031 Lublin, Poland \\ ${ }^{b}$ Institute of Nuclear Physics, Polish Academy of Sciences, \\ ul. W.E. Radzikowskiego 152, PL-31342 Kraków, Poland
}

Doi: 10.12693/APhysPolA.138.681

*e-mail: aptok@mmj.pl

\begin{abstract}
Theoretical research suggests an emergence of the Majorana bound states at the ends of nanowires. Experimental verifications of this concept have already been executed, e.g., in superconductor/semiconductor nanowire devices where interplay between a superconducting gap, spin-orbit coupling and external magnetic field allows for the creation of zero-energy bound states. Recent experiments propose a topological phase diagram by a local modification of the effective chemical potential. We discuss this possibility, using a model of an experimental system in the form of a semi-infinite $S / N$ junction. We calculate the zero-bias differential conductance $G$ in the case of the homogeneous system, as well as in the presence of the gate voltage. The relation between conductance and the effective gap in the system is investigated. We show that $G$ can reproduce the topological phase diagram in the magnetic field vs. gate voltage space of parameters.
\end{abstract}

topics: Majorana bound states, differential conductance, hybrid nanodevices

\section{Introduction}

Proposed by Kitaev in 2001, a creation of the Majorana bound states (MBS) [1] is a very attractive concept due to its non-Abelian properties [2] which can be used in performing a quantum computation via a braiding operation [3-5]. This proposal opened a period of intensive theoretical and experimental studies of the MBS [6-8].

In the Kitaev toy model [1], the MBS emerge at the end of the chain of spinless fermions with inter-site pairing. From a theoretical point of view, in the superconducting system with the spinorbit coupling, a mixture of both spin-singlet and spin-triplet Cooper pairs can be expected [9-11]. In the presence of the significantly high magnetic field, the pairing in only one branch exists [12] which is formally equivalent to a spinless $p_{x}+\mathrm{i} p_{y}$ superconductor [13].

Experimentally, this situation can be performed in the superconductor/semiconductor heterostructures [7]. In these types of systems, an interplay between an intrinsic spin-orbit coupling of a semiconductor, a superconducting gap induced in a semiconductor (by the proximity effect with superconductors $[14,15])$ and external magnetic field can lead to the emergence of the MBS [16].

An example of a practical assembly of superconductor/semiconductor nanowire devices is presented in Fig. 1a. A semiconducting nanowire (white) is partially covered by superconducting (blue) and metallic lead (orange). This is the (a)
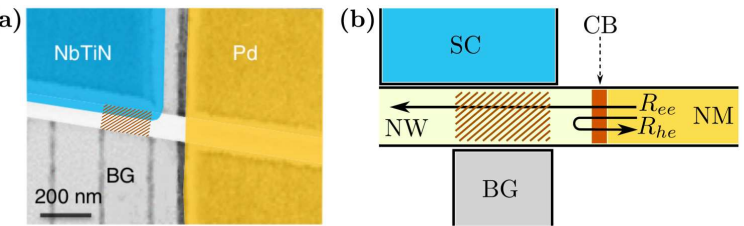

Fig. 1. (a) Scanning electron micrograph of the superconductor/semiconductor nanowire devices studied in [20]. (b) Theoretical model of the experimental system: semiconducting nanowire (NW) coupled with the normal metal (NM) lead and at the junction a Coulomb blockade (CB) exists, NW is coupled with the superconductor (SC), energy levels are shifted by the background gate (BG) voltage.

procedure generally used for obtaining the heterostructures to realization of the MBS [16-20]. Moreover, recent progress in experimental techniques of heterostructures preparation allow for the construction of additional gates [21,22]. These gates (like a background gate (BG) in Fig. 1) can be used to locally modify the effective chemical potential via the electrostatic control. Experimentally, this can be used to reproduce the topological phase diagram [19].

In the superconducting system with spin-orbit coupling, an increase of magnetic field leads to the closure of a gap and the reopening of a new topological gap. This occurs in some magnetic fields $h_{c}=\sqrt{\mu^{2}+\Delta^{2}}$ [23-25], where $\mu$ is the chemical potential (measured from the bottom of the band), 
while $\Delta$ is a superconducting gap in the absence of the magnetic field. In practice, chemical potential is fixed for given materials, while electron concentration can be changed with a gate potential [19]. In this paper, we examine this concept using the effective Bogoliubov-de Gennes tightbinding model of a one-dimensional (1D) model of the experimental system.

This paper is organized as follows: in Sect. 2, the microscopic lattice model and the technique are introduced. In sect. 3, the numerical results are described. Finally, we summarized the results in Sect. 4.

\section{Model and technique}

We focus on the single-band one-dimensional approximation which is valid when the electron occupation is low and the interband spacing is large, as compared to other energy scales in the problem [26]. The low-energy physics of the hybrid devices can be relatively well captured by this approximation [26-29]. We describe the experimental system (see Fig. 1a) using a semi-infinite model of the superconducting/normal $(\mathrm{S} / \mathrm{N})$ junction which is schematically shown in Fig. 1b. Here, the semiconducting nanowire (NW) is connected with the normal metal (NM) lead. At the connection between NW and NM, a Coulomb blockade (CB) region forms [30]. Coupling of the NW with the superconductor (SC) leads to the proximity-induced superconducting gap $\Delta$ (in calculations we assume the constant value of the gap). Additionally, in some part of NW we can modify a local chemical potential by using a background gate (BG) voltage $V_{\mathrm{BG}}$ (the brown region in Fig. 1).

In the absence of the gate, the described system can be emulated by the effective Bogoliubovde Gennes tight-binding model [31]:

$$
\begin{aligned}
& H_{0}=\sum_{i, j, \sigma}\left[-t \delta_{\langle i, j\rangle}-(\tilde{\mu}+\sigma h) \delta_{i j}\right] c_{i \sigma}^{\dagger} c_{j \sigma} \\
& +\mathrm{i} \lambda \sum_{i \sigma \sigma^{\prime}}\left(c_{i+\boldsymbol{x}, \sigma}^{\dagger} \sigma_{y}^{\sigma \sigma^{\prime}} c_{i \sigma^{\prime}}+\text { h.c. }\right) \\
& \quad+\Delta \sum_{i}\left(c_{i \uparrow}^{\dagger} c_{i \downarrow}^{\dagger}+\text { h.c. }\right)
\end{aligned}
$$

where $\langle i, j\rangle$ are the nearest-neighbor sites in the lattice, $c_{i \sigma}^{\dagger}\left(c_{i \sigma}\right)$ is the electron creation (annihilation) operator of the electron with spin $\sigma$ in site $i$. Here, $t$ is the hopping integral, $\tilde{\mu}=\mu-2 t$ is the onsite chemical potential (measured from the bottom of the band), $h$ is the Zeeman magnetic field, $\lambda$ is the Rashba spin-orbit coupling and $\sigma_{y}$ is a Pauli matrix.

We assume that the gate potential acts on the electron locally, only in some part of NW around the BG. Thus, the gate potential $V_{\mathrm{BG}}$ is given in the on-site term form:

$$
H_{\text {gate }}=\sum_{i} V_{\mathrm{BG}}(i) c_{i \sigma}^{\dagger} c_{i \sigma},
$$

where we assume $V_{\mathrm{BG}}(i) \equiv V_{\mathrm{BG}}$ for sites in the $\mathrm{BG}$ region and $V_{\mathrm{BG}}(i) \equiv 0$ otherwise. Such assumption casues that the chemical potential is locally modified by $V_{\mathrm{BG}}$. Thus, the effective chemical potential takes the following form $\tilde{\mu}_{i} \rightarrow \tilde{\mu}-V_{\mathrm{BG}}(i)$.

We calculate the conductance $G$ of the system using the $S$ matrix method [32-34]. In particular, the conductance of a $S / N$ junction is given as:

$$
G=\frac{e^{2}}{h}\left(N-R_{e e}+R_{h e}\right),
$$

where $N$ is the number of electron channels in normal lead, $R_{e h}$ is the total probability of reflection from electron to holes in normal lead, while $R_{e e}$ is the total probability of reflection from electrons to electrons in normal lead. We performed the calculations in the semi-infinity $S / N$ junction $[27,28]$, using the KWANT code [35]. In a typical situation, $G$ is quantized by $G_{0}=e^{2} / h[36]$. However, in the case of the "true" MBS measured of zero-energy, a bias peak with $G=2 G_{0}$ is expected [36, 37]. This should be treated as a sign of realization of the MBS in the system [38].

\section{Numerical results}

In our calculations, we take $\Delta / t=0.3$ and $\tilde{\mu}=0$ in the case of the homogeneous nanowire $V_{\mathrm{BG}} / t=0$. The calculation was performed for a nanowire with the length $L=100$ and 200 which corresponds to the $\mathrm{BG}$ region for $i \in(50 ; 80)$ and $(100 ; 180)$, respectively.

Firstly we discuss the general properties of the system (Fig. 2). The spectrum of the system is shown in Fig. 2a and represents characteristic features of the investigated system [39-41]. We observe a topological phase transition depending on the magnetic field (for $\mu=0$, a transition occurs at $h_{c} \simeq \Delta$ ). For the magnetic field $h=h_{c}$, a band inversion occurs [42] which is an evidence of transition from a trivial to a non-trivial phase. In the case of the system with an edge, inside a topological gap (for $h>h_{c}$ ) we observe the existence of one pair of the MBS with significantly small (close to zero) eigenenergies. States outside of the topological gap show an oscillating character which leads to its crossing [39-41]. The spectrum of the system was determined from the differential conductance $G$ (see Fig. 2b). Similarly to the previous study, an increase in $G$ intensity occurs in the crossing region of eigenvalues [43].

Figure 2 also shows two cross-sections of $G$ for two values of $h$, namely below and above $h_{c}$. For the case of $h<h_{c}$, a relatively small value of $G$ inside the trivial gap is observed. The situation is different in the case of $h>h_{c}$, where inside the topological gap, a clearly visible increase of $G$ associated with the existence of the MBS states is observed.

Further we discuss the results related to the homogeneous system. In Fig. 3a and 3c, the effective gap $2 \Delta_{\text {eff }}$ is presented. It is taken as a difference 
(a)

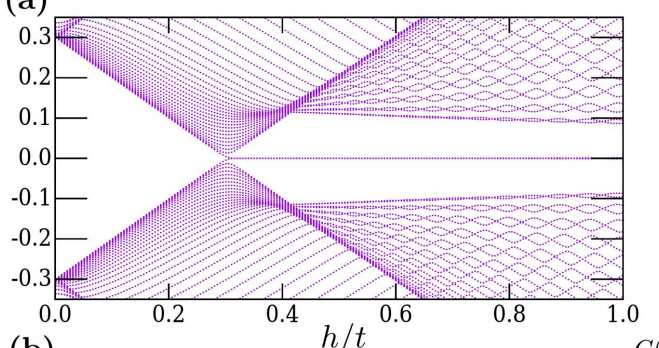

(b)

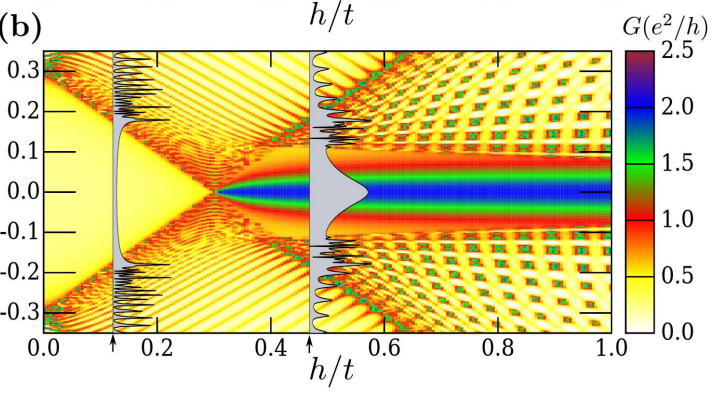

Fig. 2. (a) Spectrum of the system in the function of the magnetic field $h$. (b) The differential conductance $G$ in the function of the bias voltage and the magnetic field $h$. For two values of $h$ pointed with arrows, the contour of $G$ (black lines) is shown. Results for the homogeneous system $(\mu=0)$ with $L=100$.

between two eigenvalues of the system, nearest to the Fermi level. For the fixed chemical potential $\tilde{\mu}$, increasing the magnetic field to $h_{c}$ leads to closing of the trivial gap. Note that at $h=h_{c}$, the topological phase transition occurs and a new topological gap is reopened. However, in the finite system, for $h>h_{c}$ the exponential suppression of the gap value can be seen. It is a consequence of the existence of the MBS localized at the end of the chain, with en$\operatorname{ergy} \propto \exp (-L / \xi)$ [44], where $L$ is the system size, while $\xi$ is the correlation length. Additionally, for larger values of $h$, a typical oscillation of $2 \Delta_{\text {eff }}$ is observed due to the oscillating dependence of the energy levels around the Fermi level [45, 46]. The magnetic field dependence of $2 \Delta_{\text {eff }}$ has a strong influence on the zero-bias conductance $G$ which is shown in Fig. 3b and 3d. Independently from the length $L$ of the system, in the topological phase $\left(h>h_{c}\right)$, $G=2 G_{0}$ is observed which is an evidence of the existence of the true MBS in the system. This value exists for the broad range of parameters $h-\mu$, where the $2 \Delta_{\text {eff }}$ is significantly small, i.e., typically when $2 \Delta_{\text {eff }} / t$ is smaller than $\sim 10^{-7}$, which corresponds to the magnetic field $h / t<0.55$ and 0.75 in the case of $L=100$ and 200, respectively. Independently from this, in both cases, a boundary between the trivial and non-trivial part of the phase diagram is clearly visible and takes the form of parabolas $h_{c}=\sqrt{\mu^{2}+\Delta^{2}}$.

Further, we discuss a non-homogeneous system, in which a part of the nanowire has its effective chemical potential modified by the BG voltage. We also probe the system of different length of

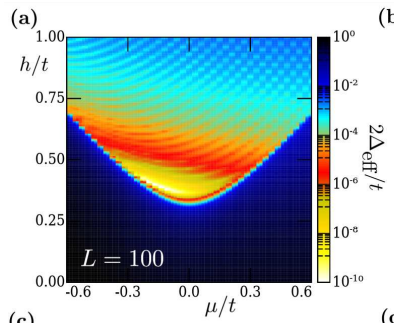

(c) ${ }_{1.00}$

$h / t$
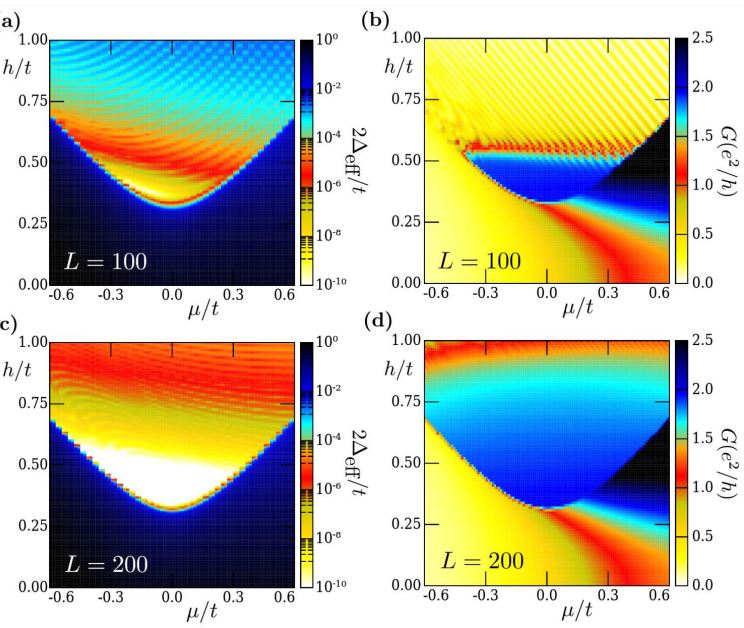

Fig. 3. Effective gap values $2 \Delta_{\text {eff }}$ given as a difference between two eigenvalues nearest to zero energy in the system of a length (a) $L=100$ and (c) $L=200$. Zero-bias differential conductance $G$. Results for the homogeneous system of a length (b) $L=100$ and (d) $L=200$.
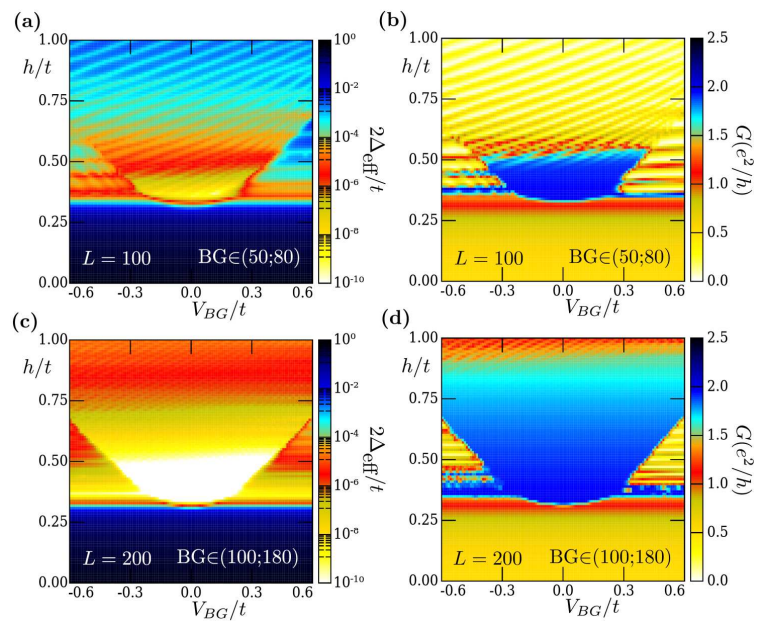

Fig. 4. (a, c) Effective gap values $2 \Delta_{\text {eff }}$ given as a difference between two eigenvalues nearest zero energy. (b, d) Zero-bias differential conductance $G$. Results for the non-homogeneous system.

the BG region. For $L=100$ and 200 we take the BG region for $i \in(50 ; 80)$ and $(100 ; 180)$, respectively. Here it should be mentioned that the length of the BG in the second case is large enough for "true" MBS to emerge in this part. For simplicity and without the loss of generality, we take $\mu=0$. As previously, in both results, $2 \Delta_{\text {eff }}$ and $G$ have a well visible parabolic-like boundaries between the trivial and non-trivial phase. However, in this case, we observed an additional horizontal line with a small value of $2 \Delta_{\text {eff }}$ with corresponding $G=2 G_{0}$. These results are independent of $V_{\mathrm{BG}}$ and are associated with an existence of the non-trivial topological phase at the non-BG region of $N W$. Moreover, its position can be modified by $\mu$. 
On the basis of the comparison of both results (see Figs. 3 and 4), we can conclude that the length of the BG region plays a similar role as the length of the homogeneous system. Indeed, in practice, the BG region can be treated as a "new" NW separated from "old" NW. In our case, the nonhomogeneous system can be represented as a few NW with different values of the chemical potential connected together.

\section{Summary}

The Majorana bound states are characterized by nearly-zero excitations whose values depend on the length of the nanowire (Fig. 2). One of signs of existence of the "true" Majorana bound states in the system can be observed as the doubled quantum of conductance $G=2 G_{0}$. These quantities can be useful in the experimental reproduction of topological phase diagrams, where the boundaries between the trivial and non-trivial topological phases are given by $h_{c}=\sqrt{\tilde{\mu}^{2}+\Delta^{2}}$.

Typically, a condition for the emergence of the topological phase depends on "internal" parameters of the system, i.e., on the chemical potential $\tilde{\mu}$ or superconducting gap $\Delta$. In this study, we show a differential conductance $G$ in the case of the homogeneous system (Fig. 3). Contemporary experimental techniques allow for local modification of $\tilde{\mu}$ by "external" parameter, i.e., a gate potential $V_{\mathrm{BG}}$. Therefore, by tuning $V_{\mathrm{BG}}$ (and independently of $\tilde{\mu}$ ), the main properties of the topological phase diagrams can be reproduced (Fig. 4). Our calculation has been performed by modeling the experimentally available system which has recently been implemented [19] to confirm a realization of the topological phase diagram in the superconductor/semiconductor nanowire devices. Both the theoretical and experimental results confirm the emergence of the topological phase in such a class of systems.

\section{Acknowledgments}

We thank Pascal Simon for an inspiring discussion. This work was supported by the National Science Centre (NCN, Poland) under grants UMO-2018/31/N/ST3/01746 (A.K.) and UMO2017/25/B/ST3/02586 (A.P.).

\section{References}

[1] A.Y. Kitaev, Phys.-Usp. 44, 131 (2001).

[2] Ch. Nayak, S.H. Simon, A. Stern, M. Freedman, S. Das Sarma, Rev. Mod. Phys. 80, 1083 (2008).

[3] J. Alicea, Y. Oreg, G. Refael, F. von Oppen, M.P.A. Fisher, Nat. Phys. 7, 412 (2011).
[4] D. Aasen, M. Hell, R.V. Mishmash, A. Higginbotham, J. Danon, M. Leijnse, T.S. Jespersen, J.A. Folk, Ch.M. Marcus, K. Flensberg, J. Alicea, Phys. Rev. X 6, 031016 (2016).

[5] A. Więckowski, M. Mierzejewski, M. Kupczyński, Phys. Rev. B 101, 014504 (2020).

[6] R. Aguado, La Rivista del Nuovo Cimento 40, 523 (2017).

[7] R.M. Lutchyn, E.P.A.M. Bakkers, L.P. Kouwenhoven, P. Krogstrup, C.M. Marcus, Y. Oreg, Nat. Rev. Mater. 3, 52 (2018).

[8] R. Pawlak, S. Hoffman, J. Klinovaja, D. Loss, E. Meyer, Prog. Part. Nucl. Phys. 107, 1 (2019).

[9] L.P. Gorkov, E.I. Rashba, Phys. Rev. Lett. 87, 037004 (2001).

[10] K. Seo, L. Han, C.A.R. Sá de Melo, Phys. Rev. A 85, 033601 (2012).

[11] A. Ptok, K. Rodriguez, K.J. Kapcia, Phys. Rev. Mater. 2, 024801 (2018).

[12] A.C. Potter, P.A. Lee, Phys. Rev. B 83, 184520 (2011).

[13] Liang Fu, C.L. Kane, Phys. Rev. Lett. 100, 096407 (2008).

[14] W. Chang, S.M. Albrecht, T.S. Jespersen, F. Kuemmeth, P. Krogstrup, J. Nygard, C.M. Marcus, Nat. Nanotech. 10, 232 (2015).

[15] Ö. Gül, H. Zhang, F.K. de Vries et al., Nano Lett. 17, 2690 (2017).

[16] V. Mourik, K. Zuo, S.M. Frolov, S.R. Plissard, E.P.A.M. Bakkers, L.P. Kouwenhoven, Science 336, 1003 (2012).

[17] M.T. Deng, S. Vaitiekénas, E.B. Hansen, J. Danon, M. Leijnse, K. Flensberg, J. Nygard, P. Krogstrup, C.M. Marcus, Science 354, 1557 (2016).

[18] H. Zhang, Ö. Gül, S. Conesa-Boj et al., Nat. Commun. 8, 16025 (2017).

[19] J. Chen, P. Yu, J. Stenger, M. Hocevar, D. Car, S.R. Plissard, E.P.A.M. Bakkers, T.D. Stanescu, S.M. Frolov, Sci. Adv. 3, e1701476 (2017).

[20] J. Chen, B.D. Woods, P. Yu, M. Hocevar, D. Car, S.R. Plissard, E.P.A.M. Bakkers, T.D. Stanescu, S.M. Frolov, Phys. Rev. Lett. 123, 107703 (2019).

[21] A. Das, Y. Ronen, Y. Most, Y. Oreg, M. Heiblum, H. Shtrikman, Nat. Phys. 8, 887 (2012).

[22] M.-T. Deng, S. Vaitiekénas, E. Prada, P. San-Jose, J. Nygard, P. Krogstrup, R. Aguado, C.M. Marcus, Phys. Rev. B 98, 085125 (2018). 
[23] M. Sato, S. Fujimoto, Phys. Rev. B 79, 094504 (2009).

[24] M. Sato, Y. Takahashi, S. Fujimoto, Phys. Rev. Lett. 103, 020401 (2009).

[25] M. Sato, Y. Takahashi, S. Fujimoto, Phys. Rev. B 82, 134521 (2010).

[26] T.D. Stanescu, S. Tewari, Phys. Rev. B 100, 155429 (2019).

[27] Ch.-X. Liu, J.D. Sau, T.D. Stanescu, S. Das Sarma, Phys. Rev. B 96, 075161 (2017).

[28] Y. Huang, H. Pan, Ch.-X. Liu, J.D. Sau, T.D. Stanescu, S. Das Sarma, Phys. Rev. B 98, 144511 (2018).

[29] H. Pan, S. Das Sarma, Phys. Rev. Res. 2, 013377 (2020).

[30] I.L. Aleiner, P.W. Brouwer, L.I. Glazman, Phys. Rep. 358, 309 (2002).

[31] A. Ptok, A. Kobiałka, T. Domański, Phys. Rev. B 96, 195430 (2017).

[32] G.B. Lesovik, I.A. Sadovskyy, Phys.-Usp. 54, 1007 (2011).

[33] A.R. Akhmerov, J.P. Dahlhaus, F. Hassler, M. Wimmer, C.W.J. Beenakker, Phys. Rev. Lett. 106, 057001 (2011).

[34] I.C. Fulga, F. Hassler, A.R. Akhmerov, C.W.J. Beenakker, Phys. Rev. B 83, 155429 (2011).
[35] Ch.W. Groth, M. Wimmer, A.R. Akhmerov, X. Waintal, New J. Phys. 16, 063065 (2014).

[36] M. Wimmer, A.R. Akhmerov, J.P. Dahlhaus, C.W.J. Beenakker, New J. Phys. 13, 053016 (2011).

[37] M. Kjaergaard, F. Nichele, H.J. Suominen et al., Nat. Commun. 7, 12841 (2016).

[38] H. Zhang, Ch.-X. Liu, S. Gazibegovic et al., Nature 556, 74 (2018).

[39] D. Sticlet, C. Bena, P. Simon, Phys. Rev. Lett. 108, 096802 (2012).

[40] B. Kiczek, A. Ptok, J. Phys.: Condens. Matter 29, 495301 (2017).

[41] A. Kobiałka, A. Ptok, Acta Phys. Pol. A 135, 64 (2018).

[42] A. Kobiałka, A. Ptok, J. Phys.: Condens. Matter 31, 185302 (2019).

[43] D. Rainis, L. Trifunovic, J. Klinovaja, D. Loss, Phys. Rev. B 87, 024515 (2013).

[44] S. Das Sarma, M. Freedman, Ch. Nayak, Npj Quantum Inf. 1, 15001 (2015).

[45] S. Das Sarma, J.D. Sau, T.D. Stanescu, Phys. Rev. B 86, 220506 (2012).

[46] S.D. Escribano, A. Levy Yeyati, E. Prada, Beilstein J. Nanotechnol. 9, 2171 (2018). 